\title{
Avaliação do processo da assistência nutricional no pré-natal em sete unidades de saúde da família do Município do Rio de Janeiro
}

\author{
Evaluation of the prenatal nutritional care process \\ in seven family health units in the city of Rio de Janeiro
}

Roberta Pereira Niquini ${ }^{1}$

Sonia Azevedo Bittencourt ${ }^{2}$

Elisa Maria de Aquino Lacerda ${ }^{3}$

Cláudia Saunders ${ }^{3}$

Maria do Carmo Leal ${ }^{2}$

${ }^{1}$ Programa de PósGraduação em

Epidemiologia em Saúde Pública, Escola Nacional de Saúde Pública Sergio Arouca (ENSP), Fundação Oswaldo Cruz (Fiocruz). Rua Leopoldo Bulhões 1480/8 Manguinhos. 21041-210

Rio de Janeiro RJ. robertaniquini@gmail.com

${ }^{2}$ Departamento de Epidemiologia e Métodos Quantitativos em Saúde, Escola Nacional de Saúde Pública Sergio Arouca (ENSP), Fundação Oswaldo Cruz (Fiocruz).

${ }^{3}$ Departamento de Nutrição e Dietética, Instituto de

Nutrição Josué de Castro,

Universidade Federal do Rio de Janeiro.
Abstract Nutritional care is of great importance in the prenatal period and the family health teams play a significant role in expanding the coverage of prenatal care. In this manner, the scope of this study was to evaluate the prenatal nutritional care process in seven family health units in the city of Rio de Janeiro. In 2008, a cross-sectional study was conducted and 230 pregnant women were interviewed and copies of their prenatal cards were obtained. The compliance of the process with the pre-established norms and criteria of the Ministry of Health was evaluated. Measurement and recording of blood pressure and weight and prescription of supplements and blood tests on the prenatal card are established steps in routine prenatal care. However, the results indicated that there was under-recording of stature, initial weight, edema, BMI by gestational age and laboratory tests results on the prenatal card. A lack of specific instruction on adequate use of the iron supplement, food consumption and weight gain was observed. The results indicated a pressing need for prenatal nutritional care and revealed deficiencies in this process, stressing the importance of minimum training for the health teams and the implementation of Family Health Support Centers.

Key words Prenatal care, Prenatal nutrition, Health evaluation, Process assessment, Family Health Program
Resumo A assistência nutricional tem grande relevância no pré-natal e as equipes de saúde da família têm papel importante na ampliação da cobertura do cuidado pré-natal. Desta forma, este estudo teve o objetivo de avaliar o processo da assistência nutricional no pré-natal em sete unidades de saúde da família do Município do Rio de Janeiro. Um estudo transversal foi conduzido em 2008 e foram entrevistadas 230 gestantes e obtidas as cópias dos cartões de pré-natal. Avaliou-se a conformidade do processo com critérios e normas pré-determinados pelo Ministério da Saúde. Os resultados indicaram que a aferição e o registro no cartão de pré-natal da pressão arterial e do peso, bem como a prescrição de suplementos e exames de sangue estão estabelecidos como rotinas do pré-natal. Também indicaram que, no cartão, há sub-registro de: estatura, peso inicial, edema, IMC por semana gestacional e resultados de exames. Verificou-se a carência de orientações específicas sobre utilização do sulfato ferroso, consumo de alimentos e ganho de peso. Os resultados revelaram uma grande necessidade de assistência nutricional e deficiências no seu processo, o que aponta para a importância do treinamento da equipe mínima e da implantação dos Núcleos de Apoio à Saúde da Família.

Palavras-chave Assistência pré-natal, Nutrição pré-natal, Avaliação em saúde, Avaliação de Processos, Programa Saúde da Família 


\section{Introdução}

Os estudos realizados com gestantes em unidades do Sistema Único de Saúde (SUS) e com mulheres em idade reprodutiva em nível domiciliar, sejam os de abrangência nacional ou os representativos de demandas restritas, revelam uma expressiva quantidade de mulheres com desvio ponderal pré-gestacional e/ou ganho de peso gestacional excessivo ou insuficiente ${ }^{1-3}$, bem como com anemia ${ }^{1,3}$, deficiência de vitamina $\mathrm{A}^{1,3}$, consumo inadequado de nutrientes ${ }^{4}$, síndromes hipertensivas $^{1,3}$ e diabetes ${ }^{1,3}$.

A grande demanda por assistência nutricional no pré-natal, a associação do estado nutricional pré-gestacional e gestacional com desfechos para a mulher e para o recém-nascido ${ }^{3,5-7}$, bem como a associação do recebimento de assistência nutricional com a melhora no consumo de energia e nutrientes ${ }^{8}$, reforçam a importância dessa assistência no pré-natal.

Apesar de ser verificada a ampliação na cobertura da atenção pré-natal no Brasil $^{1}$ e a importância das equipes de saúde da família nessa extensão ${ }^{9}$, é evidente a persistência de problemas associados à assistência oferecida. Tal comprometimento pode ser destacado pela predominância das causas obstétricas diretas no padrão de mortalidade materna no Brasil ${ }^{10}$ e pelo fato de apenas um pequeno percentual de mulheres receber as atividades assistenciais mínimas recomendadas pelo Programa de Humanização no Pré-natal e Nascimento ${ }^{11}$, o que indica que a realização dessas atividades preconizadas é o maior desafio na assistência pré-natal e aponta para a necessidade de permanentes avaliações com o objetivo de melhorar a qualidade da mesma.

Destaca-se que grande parte dos estudos que avaliam a qualidade da assistência nutricional no pré-natal, revela dados com dimensões parcialmente incompletas, que compreendem: realização de pesagem ${ }^{11-14}$, aferição de pressão arterial ${ }^{11-14}$, solicitação de hemograma ${ }^{11-15}$ e de glicemia de jejum $^{11-13}$.

Verifica-se uma menor divulgação de estudos que avaliam a aferição de estatura ${ }^{12}$, a verificação de edema ${ }^{11,14}$, a oferta de orientação alimentar ${ }^{12,15}$, a utilização de sulfato ferroso ${ }^{15}$ e de ácido fólico ${ }^{16}$ e o emprego do gráfico do estado nutricional ${ }^{11,14}$.

Tendo em vista a relevância da assistência nutricional no pré-natal, a carência de estudos que avaliem as várias dimensões do seu processo e a importância das equipes de saúde da família na ampliação da cobertura da assistência pré-natal, este estudo objetiva avaliar a conformidade do processo da assistência nutricional oferecida durante o pré-natal em sete unidades de saúde da família do Município do Rio de Janeiro, segundo as recomendações do Ministério da Saúde.

\section{Método}

Para a avaliação do processo da assistência nutricional no pré-natal utilizou-se como referencial teórico o modelo desenvolvido por Donabedian ${ }^{17}$ de avaliação dos serviços de saúde. Foi realizada uma avaliação normativa ${ }^{18}$ e formativa ${ }^{19}$ do processo da atenção nutricional no pré-natal. Essa avaliação consistiu na verificação da conformidade das atividades de assistência nutricional oferecidas durante o pré-natal, com critérios e normas pré-determinados pelo Ministério da Saúde ${ }^{20-22}$, escolhidos por serem entendidos como padrões mínimos esperados para a assistência de qualidade.

A amostra deste estudo buscou contemplar unidades de saúde da família nas áreas programáticas da cidade do Rio de Janeiro com maior expansão da estratégia de saúde da família, que melhor atendessem aos seguintes critérios: (1) unidades implantadas até seis meses antes do início da coleta de dados, (2) com mais de três equipes de saúde da família, (3) não localizadas em área de alta violência. Conforme descrito por Niquini et al. ${ }^{23}$, obteve-se uma amostra de sete unidades de saúde da família (duas unidades nas AP 3.1 e 5.1 e uma nas AP 3.3, 4.0 e 5.2).

Para o cálculo da amostra de gestantes foram utilizados os parâmetros nível de significância de 5\%, proporção de adequação da assistência pré-natal no município do Rio de Janeiro de $50 \%$, margem de erro de $5,2 \%$, sendo que o valor sofreu correção para a população finita, totalizando 230 entrevistas, as quais foram divididas de forma proporcional ao número mensal médio de consultas em cada unidade. As mulheres, com qualquer idade gestacional, foram selecionadas sistematicamente nas unidades de saúde, segundo ordem de saída das consultas de prénatal. Foi realizada a reposição das recusas $(\mathrm{n}=$ 2) na amostra.

Para a coleta de dados, realizada no período de janeiro a agosto de 2008, foi utilizada a cópia do cartão de pré-natal das gestantes e um instrumento de entrevista com elas, desenvolvido para verificar características demográficas, socioeconômicas e obstétricas e avaliar a conformidade do processo da assistência nutricional recebida. 
O instrumento de entrevista foi pré-testado e então realizado o estudo piloto. A equipe de campo foi constituída de seis supervisores e 36 entrevistadores treinados. Cada instrumento, após o preenchimento, passou por três etapas de revisão e por dupla digitação no programa Access.

Para a análise dos dados, inicialmente foi apresentada a frequência absoluta e relativa de gestantes entrevistadas de acordo com as variáveis demográficas, socioeconômicas e obstétricas (idade, raça/cor, escolaridade, situação de trabalho, situação conjugal, número de pessoas no domicílio, paridade, recebimento do benefício bolsa família e indicador de bens). Esse último foi construído com base em informações sobre a presença de dez bens para cada gestante da amostra, como descrito por Niquini et al. ${ }^{24}$.

Foi calculada, para cada gestante, a IG (idade gestacional em semanas completas) no início do pré-natal e no momento da consulta em que foi feita a entrevista. Para o cálculo da IG foi utilizada, em ordem de preferência, a data da última menstruação (DUM) anotada no cartão, a DUM referida pela mulher na data da entrevista e a informação da ultrassonografia (USG) anotada no cartão. Na ausência da informação sobre IG, as gestantes foram excluídas da análise. Para testar se as mulheres sem informação sobre IG e as com informação diferiam com relação à idade $\mathrm{e}$ à escolaridade, foi utilizado o teste não paramétrico de Mann-Whitney.

Obedecendo-se às recomendações do Ministério da Saúde de que seja realizada a primeira consulta de pré-natal com até 120 dias de gestação, duas consultas no segundo trimestre de gestação e três no terceiro ${ }^{20}$, delimitou-se, para um início precoce do pré-natal, que a primeira consulta deveria ser realizada com menos de $17 \mathrm{se}$ manas de gestação, a segunda com 17 a 22 semanas, a terceira com 23 a 28 semanas, a quarta com 29 a 34 semanas, a quinta com 35 a 37 semanas e a sexta com 38 ou mais semanas.

Como apontado no Quadro 1, a periodicidade dos procedimentos analisados nesse estudo foi então categorizada em A (que devem ser realizados em todas as consultas), B (que devem ser realizados na primeira consulta), C (realizados a partir da $17^{a}$ semana de gestação), D (realizados a partir da $23^{a}$ semana de gestação) e E (realizados a partir da 29a semana de gestação), apresentada segundo o início do pré-natal precoce (com menos de 17 semanas de gestação) ou tardio (com 17 ou mais semanas de gestação). Os procedimentos foram classificados também segundo a fonte de informação (cartão de pré-natal e entre- vista com gestantes) e agrupados em quatro categorias de acordo com o tipo de procedimento, em: (I) aferição e registro das medidas antropométricas, da pressão arterial e registro de presença de edema, (II) solicitação de exames e registro dos resultados, (III) suplementação de vitaminas e minerais e (IV) orientações alimentares e sobre a adequação do ganho de peso.

Os registros de peso, pressão arterial, edema e marcação do gráfico de Índice de Massa Corporal (IMC) por semana gestacional no cartão foram mensurados como razão desses registros por número de consultas e foram considerados em conformidade quando a razão apresentou valores entre 0,75 e 1,0. Para as orientações alimentares, foi considerado em conformidade ter recebido mais de $75 \%$ das oito orientações avaliadas. Os demais procedimentos foram avaliados de forma dicotômica.

A verificação da adequação da orientação recebida sobre o ganho de peso entre a penúltima e a última consulta foi feita para gestantes com duas ou mais consultas, que tiveram a penúltima após a 13a semana de gestação. Nesta etapa, foram excluídas as gestantes com gestação gemelar e que apresentaram edema em alguma das consultas de pré-natal, segundo informações presentes no cartão de pré-natal.

Para a verificação da adequação do ganho de peso, foi realizado o cálculo do IMC inicial de cada gestante. Para tal, foram utilizadas as médias dos valores de estatura referido pela gestante na entrevista e registrado no cartão (coeficiente de correlação intraclasse (ICC) 0,81 ; IC $95 \%$ de $0,53$ a 0,96$)$ e as médias dos valores de peso prégestacional referido pela gestante na entrevista e registrado no cartão (ICC 0,95; IC 95\% 0,92 a $0,98)$. Quando só uma fonte de informação estava disponível, a mesma foi utilizada. O estado nutricional inicial, segundo o IMC pré-gestacional, foi classificado utilizando o critério indicado pela Organização Mundial da Saúde (OMS) para adolescentes e adultas ${ }^{7}$. Quando o IMC pré-gestacional não estava disponível, foi utilizado o IMC na primeira consulta de pré-natal, e o estado nutricional foi classificado segundo a $\mathrm{OMS}^{7}$, quando IG na primeira consulta $<14$ semanas e segundo a tabela de IMC por semana de gestação $0^{25}$, quando $>14$ semanas.

Foram estimados valores máximos e mínimos de ganho de peso semanal para cada gestante, segundo os limites superior e inferior de ganho de peso gestacional total fornecidos pelo IOM para cada categoria de estado nutricional inicial ${ }^{26}$. A primeira etapa do cálculo consistiu 
Quadro 1. Procedimentos de assistência nutricional no pré-natal, segundo periodicidade, tipo e fontes de informação. Rio de Janeiro (RJ), 2007.

\begin{tabular}{|c|c|c|c|c|c|}
\hline \multirow[b]{2}{*}{ Procedimentos } & \multicolumn{2}{|c|}{ Periodicidade } & \multirow[b]{2}{*}{ Tipo } & \multicolumn{2}{|r|}{ Fonte } \\
\hline & $\begin{array}{l}\text { Início do } \mathrm{PN} \\
<17 \text { sem }\end{array}$ & $\begin{array}{l}\text { Início do } \mathrm{PN} \\
>17 \mathrm{sem}\end{array}$ & & $\begin{array}{l}\text { Cartão de } \\
\text { Pré-natal }\end{array}$ & $\begin{array}{c}\text { Entrevista } \\
\text { com gestantes }\end{array}$ \\
\hline 1) Registro/aferição do peso atual & A & $\mathrm{A}$ & I & & \\
\hline 2) Registro/aferição da pressão arterial & A & A & $\mathrm{I}$ & & \\
\hline $\begin{array}{l}\text { 3) Registro de verificação de } \\
\text { presença de edema }\end{array}$ & A & A & $\mathrm{I}$ & & \\
\hline $\begin{array}{l}\text { 4) Marcação no gráfico de avaliação } \\
\text { do estado nutricional }\end{array}$ & A & A & I & & \\
\hline 5) Registro de peso pré-gestacional & B & B & I & & \\
\hline 6) Registro/aferição de estatura & B & B & I & & \\
\hline 7) Solicitação de exame de sangue & B & B & II & & \\
\hline $\begin{array}{l}\text { 8) Orientações alimentares: (1) } \\
\text { comer mais frutas e legumes; }(2) \\
\text { beber mais leite, iogurte ou queijo; } \\
\text { (3) beber bastante água; (4) comer } \\
\text { menos sal; (5) comer mais feijão; (6) } \\
\text { comer fígado } 1 \text { vez por semana; ( } 7) \\
\text { as quantidades e os alimentos que } \\
\text { deveria comer em cada refeição; ( } 8) \\
\text { se recebeu alguma orientação por } \\
\text { escrito sobre alimentação na } \\
\text { gravidez. }\end{array}$ & $\mathrm{C}$ & $\mathrm{D}$ & IV & & \\
\hline 9) Registro /prescrição de ácido fólico & $\mathrm{D}$ & $\mathrm{D}$ & III & & \\
\hline $\begin{array}{l}\text { 10) Registro/prescrição de sulfato } \\
\text { ferroso }\end{array}$ & $\mathrm{D}$ & $\mathrm{D}$ & III & & \\
\hline $\begin{array}{l}\text { 11) Orientação para o uso do sulfato } \\
\text { ferroso }\end{array}$ & $\mathrm{D}$ & $\mathrm{D}$ & III & & \\
\hline $\begin{array}{l}\text { 12) Orientação correta sobre ganho } \\
\text { de peso }\end{array}$ & $\mathrm{D}$ & $\mathrm{D}$ & IV & & \\
\hline $\begin{array}{l}\text { 13) Registro do resultado do } 1^{\circ} \\
\text { exame de Hemoglobina }\end{array}$ & $\mathrm{D}$ & $\mathrm{E}$ & II & & \\
\hline $\begin{array}{l}\text { 14) Registro do resultado do } 1^{\circ} \\
\text { exame de Glicemia de Jejum }\end{array}$ & $\mathrm{D}$ & $\mathrm{E}$ & II & & \\
\hline
\end{tabular}

Notas: PN= pré-natal; Sem = semanas; Periodicidade: A - em todas as consultas, B - na primeira consulta, C - a partir da 17a semana de gestação, D - a partir da 23a semana de gestação, E - a partir da $29^{a}$ semana de gestação; Tipos de procedimentos: I - Aferição e registro das medidas antropométricas, da pressão arterial e registro de presença de edema, II - Solicitação de exames e registro dos resultados, III - Suplementação de vitaminas e minerais, IV - Orientações alimentares e sobre a adequação do ganho de peso. 
em subtrair o ganho de peso da gestante até a penúltima consulta de gestação do limite superior e do limite inferior do ganho total recomendado, enquanto a segunda etapa versou em dividir os dois resultados encontrados na primeira etapa pelo tempo restante de gestação, contando a partir da penúltima consulta.

Como para obesas não existem limites máximos e mínimos de ganho de peso total, o ganho total estipulado para obesas $(7 \mathrm{~kg}$ ) foi considerado como ganho total mínimo e o ganho total máximo foi calculado multiplicando-se o ganho de peso médio semanal para o segundo e terceiro trimestres para obesas $(0,3 \mathrm{~kg} / \mathrm{semana})$ por 27 semanas, totalizando $8,1 \mathrm{~kg}$.

Para gestantes cujo valor de ganho de peso mínimo/semanal estimado foi inferior ao modesto recomendado $(0,250 \mathrm{~kg} / \mathrm{semana}$ para gestantes com IMC inicial normal e de baixo peso e de $0,125 \mathrm{~kg} / \mathrm{semana}$ para gestantes com sobrepeso e obesidade $)^{27,28}$, os valores modestos foram utilizados como mínimos. Quando o valor de ganho de peso máximo/semanal estimado foi inferior ou igual ao modesto recomendado, utilizaram-se como valores máximos os valores modestos recomendados acrescidos de $20 \%{ }^{28}$. No caso de ausência de informação sobre o peso antes de 14 semanas de gestação, a faixa de adequação do ganho de peso foi construída com o ganho de peso semanal médio recomendado para o $2^{\circ}$ e $3^{\circ}$ trimestres, segundo categoria de estado nutricional inicial ${ }^{20,26} \pm 20 \%{ }^{28}$. Os valores máximos semanais estimados para a faixa de adequação do ganho de peso individual não ultrapassaram $0,750 \mathrm{~kg} /$ semana $^{27}$.

Com base nos limites de ganho de peso calculados para cada gestante foi verificada a adequação da orientação recebida sobre o ganho de peso.

Para avaliar se existe diferença da proporção de conformidade de cada procedimento entre os grupos divididos segundo variáveis demográficas, socioeconômicas e obstétricas das gestantes, foi utilizado o teste do qui-quadrado de Pearson, com correção de Yates ou o teste exato de Fisher, quando as condições para a utilização do teste qui-quadrado não foram verificadas. Os resultados foram considerados estatisticamente significantes para um valor de $\mathrm{p}<0,05$.

A estatística Kappa ajustada ${ }^{29}$ foi utilizada para verificar a confiabilidade entre as informações obtidas através do cartão de pré-natal e da entrevista com as gestantes, sendo a concordância das duas fontes de informação classificada segundo Landis e $\mathrm{Koch}^{30}$. As análises foram realizadas no software R versão 2.7.0.
A pesquisa foi conduzida dentro dos padrões exigidos pela Declaração de Helsinque e aprovada pelos Comitês de Ética em Pesquisa da Escola Nacional de Saúde Pública Sergio Arouca (ENSP)/ Fiocruz e da Secretaria Municipal de Saúde do Rio de Janeiro. Não há conflito de interesse em relação aos métodos utilizados como parte da investigação ou interesse financeiro dos pesquisadores.

\section{Resultados}

Nas sete unidades de saúde da família selecionadas para o estudo foram entrevistadas 230 gestantes e 214 tinham informação sobre a IG. As mulheres com e sem informação sobre IG (7\%) não diferiram com relação à idade e anos de estudo $(\mathrm{p}>0,05)$.

Na Tabela 1, apresenta-se a distribuição das gestantes que tinham informação sobre IG, de acordo com as variáveis demográficas, socioeconômicas e obstétricas. A amostra de gestantes era composta, em sua maioria, de mulheres adultas $(74,8 \%)$, pardas $(57,9 \%)$, com 8 ou mais anos de estudo $(57,5 \%)$, sem trabalho remunerado $(76,2 \%)$, que viviam com companheiro $(81,8 \%)$ e que moravam com até mais duas pessoas $(51,9 \%)$.

O valor do indicador de bens variou de zero a cinco e 52,8\% das gestantes apresentaram indicador de bens maior que um. Destaca-se que $84,6 \%(n=181)$ das gestantes relataram não serem beneficiárias do programa bolsa família, embora 39 dessas tenham informado uma renda per capita menor que R\$120,00.

Entre as 214 gestantes entrevistadas, $61,2 \%$ já tinham um ou mais filhos e $71 \%$ tiveram início precoce do pré-natal.

Como pode ser observado na Tabela 2, 87,4\% das gestantes tiveram a razão de registros de peso atual no cartão de pré-natal por número de consultas em conformidade, valor que foi de 93,9\% para a razão de registro de pressão arterial por número de consultas.

A razão de registros de edema por números de consultas estava em conformidade para $17,1 \%$ $(\mathrm{n}=20)$ das gestantes que portavam cartões com campos para registro de edema $(n=117)$.

O modelo de cartão atual do Ministério da Saúde (2005), único que possui o gráfico de IMC/ semana de gestação, era portado por 97 gestantes, sendo que $90,7 \%$ não tinham qualquer marcação no gráfico e apenas 3,1\% possuíam conformidade da razão do número de marcações no gráfico por número de consultas. 
Tabela 1. Distribuição das gestantes com informação sobre idade gestacional de acordo com as variáveis demográficas, socioeconômicas e obstétricas. Rio de Janeiro - RJ, 2007 ( $n=214)$.

\begin{tabular}{|c|c|c|}
\hline Variáveis & $\mathbf{n}$ & $\%$ \\
\hline \multicolumn{3}{|l|}{ Idade } \\
\hline$\geq 20$ anos & 160 & 74,8 \\
\hline$<20$ anos & 54 & 25,2 \\
\hline \multicolumn{3}{|l|}{ Cor/Raça } \\
\hline Branca & 47 & 22,0 \\
\hline Preta & 40 & 18,7 \\
\hline Pardas & 124 & 57,9 \\
\hline Amarelas e Indígenas & 3 & 1,4 \\
\hline \multicolumn{3}{|l|}{ Escolaridade } \\
\hline Menos de 8 anos de estudo & 91 & 42,5 \\
\hline 8 ou mais anos de estudo & 123 & 57,5 \\
\hline \multicolumn{3}{|l|}{ Situação de Trabalho } \\
\hline Com trabalho remunerado & 51 & 23,8 \\
\hline Sem trabalho remunerado & 163 & 76,2 \\
\hline \multicolumn{3}{|l|}{ Situação Conjugal } \\
\hline Vive com companheiro & 175 & 81,8 \\
\hline Não vive com companheiro & 39 & 18,2 \\
\hline \multicolumn{3}{|l|}{ No de pessoas no domicílio } \\
\hline 1 a 3 & 111 & 51,9 \\
\hline 4 ou mais & 103 & 48,1 \\
\hline \multicolumn{3}{|l|}{ Indicador de Bens } \\
\hline$\leq 1$ & 101 & 47,2 \\
\hline$>1$ & 113 & 52,8 \\
\hline \multicolumn{3}{|l|}{ Recebe Bolsa Família } \\
\hline Sim & 33 & 15,4 \\
\hline Não & 181 & 84,6 \\
\hline \multicolumn{3}{|l|}{ Nuliparidade } \\
\hline Não & 131 & 61,2 \\
\hline Sim & 83 & 38,8 \\
\hline \multicolumn{3}{|c|}{ Idade Gestacional no Início do Pré-Natal } \\
\hline$<17$ semanas & 152 & 71,0 \\
\hline$\geq 17$ semanas & 62 & 29,0 \\
\hline \multicolumn{3}{|l|}{ Idade Gestacional na Entrevista } \\
\hline$<17$ semanas & 41 & 19,2 \\
\hline 17 a 22 semanas & 33 & 15,4 \\
\hline 23 a 28 semanas & 39 & 18,2 \\
\hline 29 a 34 semanas & 46 & 21,5 \\
\hline 35 a 37 semanas & 33 & 15,4 \\
\hline$\geq 38$ semanas & 22 & 10,3 \\
\hline
\end{tabular}

O registro de peso pré-gestacional foi feito em $65,9 \%$ dos cartões de pré-natal. Entre as gestantes que possuíam cartões com campos para registro de estatura $(\mathrm{n}=194), 57,7 \%$ apresentaram registro.

Ao considerar apenas as gestantes com 23 ou mais semanas de gestação na data da entrevista $(n=140)$, o registro no cartão de pré-natal da prescrição de sulfato ferroso e de ácido fólico ocorreu para $16,4 \%$.
Tabela 2. Conformidade dos procedimentos de assistência nutricional no pré-natal, segundo registros do cartão de pré-natal. Rio de Janeiro (RJ), 2007.

\begin{tabular}{lcc}
\hline \multicolumn{1}{c}{ Procedimentos } & $\begin{array}{c}\text { Conformidade } \\
\text { \% (n) }\end{array}$ & Total \\
\hline $\begin{array}{l}\text { Razão de registros do peso } \\
\text { atual por no de consultas }\end{array}$ & $87,4(187)$ & 214 \\
$\geq 0,75$ & & \\
$\begin{array}{l}\text { Razão de registros de } \\
\text { pressão arterial por no de }\end{array}$ & $93,9(201)$ & 214 \\
consultas $\geq 0,75$ & & \\
$\begin{array}{l}\text { Razão de registros de } \\
\text { presença de edema por }\end{array}$ & $17,1(20)$ & 117 \\
$\begin{array}{l}\text { no de consultas } \geq 0,75 \\
\text { Razão de marcações no } \\
\text { gráfico de avaliação do }\end{array}$ & $3,1(3)$ & \\
estado nutricional por & & \\
no de consultas $\geq 0,75$ & & \\
$\begin{array}{l}\text { Registro de peso } \\
\text { pré-gestacional }\end{array}$ & $65,9(141)$ & 214 \\
$\begin{array}{l}\text { Registro de estatura } \\
\text { Registro de prescrição de } \\
\text { sulfato ferroso }\end{array}$ & $16,4(23)$ & 140 \\
$\begin{array}{l}\text { Registro de prescrição de } \\
\text { ácido fólico }\end{array}$ & $16,4(23)$ & 140 \\
$\begin{array}{l}\text { Registro do resultado do } \\
\text { exame de hemoglobina } \\
\text { Registro do resultado do } \\
\text { exame de glicemia de jejum }\end{array}$ & $67,8(82)$ & 121 \\
\hline
\end{tabular}

O registro do primeiro exame de hemoglobina foi encontrado em $67,8 \%$ dos cartões avaliados $(n=121)$ e o de glicemia de jejum em $78,5 \%$.

Ao estratificar as gestantes segundo grupo de idade, de escolaridade, de situação de trabalho, de situação conjugal e de recebimento de bolsa família, não foi verificada diferença na proporção de conformidade para nenhum dos procedimentos, segundo registro no cartão de pré-natal, ao nível de 5\% de significância.

Estratificando as gestantes segundo o número de pessoas no domicílio, verificou-se diferença significativa da proporção de conformidade entre os grupos para os dois procedimentos: registro de peso pré-gestacional no cartão $(p=0,023)$ e registro de estatura no cartão $(p=0,023)$. A conformidade desses procedimentos foi de, respectivamente, $73 \%$ e $65,7 \%$ para as gestantes que moram com até mais duas pessoas e de $58,2 \%$ e $49,5 \%$ para as que moram com três pessoas ou mais.

A diferença significativa da proporção de conformidade para o procedimento "registro de es- 
tatura no cartão", também foi observada ao estratificar as gestantes segundo paridade $(\mathrm{p}=$ 0,022 ), verificando-se uma conformidade de $68 \%$ para as nulíparas e 51,3\% para as que já tiveram um ou mais partos.

Ao estratificar as gestantes em grupos segundo categorias do indicador de bens, verifica-se diferença significativa da proporção de conformidade entre os grupos para o procedimento "registro do $1^{\circ}$ exame de glicemia" ( $\left.\mathrm{p}=0,037\right)$, com $72,7 \%$ entre as com indicador de bens menor ou igual a um e $88,1 \%$ para as com indicador de bens maior que um.

Como pode ser observado na Tabela 3, segundo informações obtidas através das entrevistas, 92,1\% das gestantes afirmaram que foram pesadas em todas as consultas de pré-natal e $60,7 \%$ responderam que sua estatura foi aferida no pré-natal.

A questão sobre a aferição da pressão arterial foi respondida por 200 gestantes, das quais $98,5 \%$ afirmaram que tiveram a pressão arterial aferida em todas as consultas. Todas as gestantes afirmaram que o exame de sangue foi solicitado durante o pré-natal.

Entre as gestantes para as quais foi avaliado o recebimento de orientações alimentares, 31,5\% referiram o recebimento de 7 a 8 das orientações pesquisadas, 38,9\% relataram terem sido orientadas quanto às quantidades e os alimentos que deveriam comer em cada refeição e 14,8\% rece- beram alguma orientação por escrito sobre alimentação na gravidez.

O recebimento de alguma forma de orientação sobre alimentação foi referido por $92 \%$ ( $n=$ 149) das gestantes avaliadas para esse critério (n $=162)$, das quais, $65,1 \%(\mathrm{n}=97)$ afirmaram que quem fez a orientação foi o enfermeiro, 24,2\% (n = 36) o médico, 8,7\% ( $\mathrm{n}=13)$ o médico e o enfermeiro, $1,3 \%(\mathrm{n}=2)$ o médico, o enfermeiro e o nutricionista e $0,7 \%(\mathrm{n}=1)$ o nutricionista. Entre elas, 97,4\% $(n=145)$ informaram que receberam orientação na consulta.

Do total de gestantes com informações disponíveis para o cálculo do IMC inicial $(\mathrm{n}=171)$, $6,4 \%(n=11)$ foram classificadas com baixo peso, $62 \%(n=106)$ como eutróficas, $19,3 \%(n=33)$ como sobrepeso e $12,3 \%(\mathrm{n}=21)$ como obesas.

Ao considerar as gestantes que tinham informação sobre o IMC inicial, 127 destas já deveriam ter recebido orientação nutricional, segundo o critério utilizado neste estudo. Para as que estavam com o IMC inicial inadequado (51/127), $47,1 \%(n=24)$ receberam orientação sobre as quantidades e os alimentos que deveriam comer em cada refeição e 19,6\% $(n=10)$ alguma orientação por escrito sobre alimentação na gravidez. Entre as que estavam com o IMC inicial adequado (76/127), esses valores foram de 36,8\% ( $\mathrm{n}=$ 28) e $15,8 \%(n=12)$. Não foi encontrada diferença estatisticamente significativa, ao nível de $5 \%$, entre as proporções de recebimento de orien-

Tabela 3. Conformidade dos procedimentos de assistência nutricional no pré-natal, segundo entrevista com a gestante. Rio de Janeiro - RJ, 2007.

\begin{tabular}{lrr}
\hline \multicolumn{1}{c}{ Procedimentos } & Conformidade \% (n) & Total \\
\hline Aferição do peso atual & $92,1(197)$ & 214 \\
Aferição da pressão arterial & $98,5(197)$ & 200 \\
Aferição de estatura & $60,7(130)$ & 214 \\
Solicitação de exame de sangue & $100,0(214)$ & 214 \\
Recebimento de 7 a 8 orientações alimentares & $31,5(51)$ & 162 \\
Recebimento de orientação sobre: & & \\
comer mais frutas e legumes & $88,3(143)$ & 162 \\
beber mais leite, iogurte ou queijo & $72,2(117)$ & 162 \\
beber bastante água & $90,1(146)$ & 162 \\
comer menos sal & $77,8(126)$ & 162 \\
comer mais feijão & $74,1(120)$ & 162 \\
comer fígado 1 vez por semana & $56,8(92)$ & 162 \\
as quantidades e os alimentos que deveria comer em cada refeição & $38,9(63)$ & 162 \\
orientação por escrito sobre alimentação na gravidez & $14,8(24)$ & 162 \\
Orientação correta sobre ganho de peso & $28,3(26)$ & 92 \\
Prescrição de sulfato ferroso & $98,6(138)$ & 140 \\
Prescrição de ácido fólico & $81,2(112)$ & 138 \\
Orientação para o uso do sulfato ferroso & $30,0(42)$ & 140 \\
\hline
\end{tabular}


tações entre os grupos com IMC inicial adequado e inadequado.

Para as 121 gestantes que seriam avaliadas quanto à adequação da orientação recebida sobre o ganho de peso da penúltima para a última consulta, segundo o critério utilizado neste estudo, 99 tinham informações disponíveis para o cálculo, nenhuma tinha gestação gemelar, entretanto, sete apresentaram edema, sendo excluídas dessa análise. Ao avaliar as 92 gestantes, verificou-se que $28,3 \%(n=26)$ receberam a orientação correta sobre o seu ganho de peso (Tabela 4), valor que foi de $21,1 \%(n=15)$ para as gestantes que tiveram ganho de peso semanal abaixo ou acima do recomendado $(\mathrm{n}=71)$ e de $52,4 \%(\mathrm{n}=$ 11) ao considerar as gestantes que tiveram ganho de peso semanal adequado $(n=21)$. A diferença entre as proporções de recebimento de orientação correta sobre o ganho de peso entre os grupos com ganho semanal adequado e inadequado foi significativa ao nível de $5 \%(\mathrm{p}=0,005)$.

$\mathrm{O}$ ganho de peso inadequado foi observado entre 71 gestantes, das quais $43,7 \%(n=31)$ receberam orientação sobre as quantidades e os alimentos que deveriam comer em cada refeição e $15,5 \%(n=11)$ receberam alguma orientação por escrito sobre alimentação na gravidez. Para as que tiveram o ganho de peso adequado $(n=21)$, esses valores foram de $52,4 \%(n=11)$ e $23,8 \%(n$ $=5)$. Não foi encontrada diferença estatisticamente significativa, ao nível de $5 \%$, entre as proporções de recebimento de orientações alimentares entre os grupos com ganho de peso inadequado e adequado.

Entre as gestantes que, na entrevista, estavam com 23 ou mais semanas de gestação $(n=140)$, $98,6 \%(n=138)$ referiram a prescrição de sulfato ferroso e $30 \%(n=42)$ receberam orientação para tomar o suplemento uma hora antes das refeições. Vale ressaltar que 70,7\% $(n=99)$ das gestantes relataram o uso do sulfato ferroso ou outro remédio com ferro.
Ao considerar as gestantes que tinham registro no cartão de pré-natal do primeiro exame de hemoglobina e que foram entrevistadas com 23 ou mais semanas de gestação $(n=82)$, entre as 13 que apresentaram anemia leve, moderada ou grave (hemoglobina menor que $11 \mathrm{~g} / \mathrm{dL}$ ), 38,5\% $(n=5)$ receberam orientação para tomar o sulfato ferroso uma hora antes das refeições, valor que foi de $24,6 \%(n=17)$ entre as não-anêmicas $(n=69)$. Ressalta-se, entretanto, que não foi verificada diferença significativa entre as proporções de orientações adequadas para uso do sulfato ferroso entre os grupos de mulheres com anemia e não anêmicas.

Para as 138 gestantes que estavam com 23 ou mais semanas de gestação e responderam à questão sobre a prescrição de ácido fólico, 81,2\% (n = 112) informaram que o mesmo foi prescrito.

Com relação às informações obtidas através de entrevista com as gestantes, ao estratificar as gestantes segundo o grupo de idade, a situação de trabalho, as situações conjugal, de paridade e de recebimento de bolsa família, não foi verificada diferença da proporção de conformidade para nenhum dos procedimentos, ao nível de $5 \%$ de significância.

Estratificando as gestantes segundo escolaridade, o único procedimento para o qual se verificou diferença significativa da proporção de conformidade entre os grupos foi "receber orientação para beber bastante água” ( $\mathrm{p}=0,037)$, a qual foi de $95,7 \%$ entre as gestantes com menos de oito anos de estudo e $85,9 \%$ entre as com oito ou mais anos.

Ao estratificar os grupos segundo o número de pessoas no domicílio, verificou-se diferença significativa da proporção de conformidade entre os grupos para o procedimento "receber orientação para comer mais frutas e legumes” ( $p=0,024)$, que foi de 93,9\% para as gestantes que moram com até mais duas pessoas e $82,5 \%$ para as gestantes que moram com três pessoas ou mais.

Tabela 4. Ganho de peso da última para a penúltima consulta de pré-natal e orientação recebida sobre o ganho de peso. Rio de Janeiro - RJ, 2007. ( $n=92)$

\begin{tabular}{lcccr}
\hline \multirow{2}{*}{$\begin{array}{c}\text { Orientação recebida } \\
\text { sobre o ganho de peso }\end{array}$} & \multicolumn{4}{c}{ Ganho de peso da última para a penúltima semana } \\
\cline { 2 - 5 } & $\begin{array}{c}\text { Abaixo do recomendado } \\
\%(\mathbf{n})\end{array}$ & $\begin{array}{c}\text { Adequado } \\
\%(\mathbf{n})\end{array}$ & $\begin{array}{c}\text { Acima do recomendado } \\
\%(\mathbf{n})\end{array}$ & $\begin{array}{c}\text { Total } \\
\%(\mathbf{n})\end{array}$ \\
\hline Não receberam orientação & $15,8(6)$ & $28,6(6)$ & $24,2(8)$ & $21,7(20)$ \\
Correta & $5,3(2)$ & $52,4(11)$ & $39,4(13)$ & $28,3(26)$ \\
Incorreta & $78,9(30)$ & $19,0(4)$ & $36,4(12)$ & $50,0(46)$ \\
Total & $100,0(38)$ & $100,0(21)$ & $100,0(33)$ & $100,0(92)$ \\
\hline
\end{tabular}


A estratificação das gestantes, segundo categorias do indicador de bens, apontou uma diferença significativa da proporção de conformidade entre os grupos para o procedimento "receber orientação por escrito sobre alimentação na gravidez" ( $p=0,025)$, com $8 \%$ entre as com indicador de bens menor ou igual a um e $20,8 \%$ para as com indicador de bens maior que um.

O valor da estatística Kappa ajustada das informações de registro/ aferição do peso atual $(0,81)$ e da pressão arterial $(0,84)$ foram quase perfeitas, enquanto a de registro/ aferição de estatura $(0,47)$ foi moderada e as de registros/prescrições de sulfato ferroso $(-0,61)$ e de ácido fólico $(-0,35)$ foram pobres.

$\mathrm{O}$ percentual de gestantes que tiveram todos os procedimentos tipo I, II, III e IV em conformidade foi de respectivamente, $0 \%, 79,9 \%, 7,1 \%$ e $13,6 \%$.

\section{Discussão}

A incorporação da pesagem e da aferição da pressão arterial na rotina do pré-natal observada é corroborada pelo estudo de Costa et al., em que $100 \%$ das gestantes relataram que peso e pressão arterial foram avaliados em todas as consultas de pré-natal ${ }^{31}$, de Carvalho e Novaes, em que mais de $90 \%$ das gestantes revelaram que essas aferições são sempre realizadas ${ }^{12}$, de Parada, no qual menos de $10 \%$ dos prontuários não tinham registro de peso e pressão arterial em todas as consultas $^{13}$ e pelo estudo de Coutinho et al., em que mais de $75 \%$ dos cartões tinham cinco ou mais registros dessas medidas ${ }^{11}$.

Não tão amplamente fixados nas rotinas de pré-natal, o registro e a aferição da estatura, apesar de terem sido mais valorizado para mulheres nulíparas, não foram condutas realizadas com maior frequência entre as gestantes adolescentes, o que preocupa devido ao crescimento que estas gestantes apresentam ao longo da gestação ${ }^{20}$. Ressalta-se que a ausência de informação sobre estatura prejudica a avaliação do estado nutricional inicial da gestante, a avaliação do ganho de peso e do estado nutricional ao longo da gestação.

A baixa valorização dada à avaliação e ao acompanhamento do estado nutricional e do ganho de peso é reforçada pelo baixo preenchimento do gráfico de IMC por semana gestacional e dos campos referentes à presença de edema, problemas já descritos em outros estudos ${ }^{11,14,31}$. O baixo preenchimento dos campos presentes no cartão de pré-natal preocupa, pois esse ins- trumento de registro tem o papel de permitir o fluxo de informações entre os serviços de saúde e o acompanhamento da evolução da gravidez, do parto e do puerpério ${ }^{20}$.

Com relação aos procedimentos tipo II, dados da Pesquisa Nacional de Demografia e Saúde de 2006 revelaram que a realização de exame de sangue no pré-natal foi referida por $94,1 \%$ das mulheres na região Sudeste ${ }^{1}$, o que está de acordo com o elevado percentual de solicitação do exame encontrado no presente estudo. Quanto aos registros do primeiro exame de hemoglobina e de glicemia de jejum no cartão de pré-natal, ressaltase que percentuais relativamente similares aos observados foram encontrados em um estudo realizado no estado de Minas Gerais ${ }^{11}$. É possível que esteja ocorrendo uma carência de registro dos resultados dos exames no cartão de pré-natal e até uma demora na realização dos mesmos.

O baixo registro observado da prescrição dos suplementos (procedimentos tipo III) pode ocorrer pelo fato deste campo não estar presente em todos os modelos dos cartões utilizados, desestimulando o preenchimento do mesmo pelos profissionais $^{23}$. A confiabilidade pobre entre as informações sobre suplementação obtidas por meio dos cartões e das entrevistas com gestantes também pode ser resultado do desconhecimento, por parte de algumas gestantes, da composição dos suplementos que fazem uso ${ }^{16}$.

Apesar de ter sido encontrada uma prevalência importante de anemia entre as mulheres com mais de 23 semanas de gestação que tinham registro do exame de hemoglobina no cartão, a mesma foi inferior à observada para gestantes atendidas em uma maternidade do Município do Rio de Janeiro $(22,9 \%)^{3}$ e para mulheres em idade reprodutiva residentes na Região Sudeste do Brasil $(28,5 \%)^{1}$, o que chama a atenção para a possível existência de gestantes com anemia não diagnosticada entre as sem registro de hemoglobina.

Com relação à oferta de orientações alimentares (procedimentos tipo IV), destaca-se que as gestantes que moravam com menos de três pessoas e as que tinham indicador de bens maior que um, tiveram um maior relato de recebimento de algumas orientações sobre o consumo de alimentos. Uma hipótese para esses achados é a de que os profissionais de saúde da família não abordem a questão da qualidade e quantidade de consumo de alimentos para algumas famílias grandes, de baixa renda, por saberem de sua dificuldade para adquirir os alimentos. Outra hipótese, é a de que as mulheres com famílias maiores e de baixa renda, não valorizem a orienta- 
ção recebida, por não terem condição de adquirir esses alimentos ou por oferecerem os mesmos, preferencialmente, para seus filhos, sugerindo uma maior fixação da orientação quando ela está inserida na realidade vivida. Vale destacar que, o perfil das mulheres entrevistadas revelou gestantes em situação de pobreza ${ }^{32}$, que afirmaram não serem beneficiárias do programa bolsa família, apontando para uma possível condição de insegurança alimentar ${ }^{1}$.

Ressalta-se que as orientações nutricionais mais específicas, como as quantidades e os alimentos que deveria comer em cada refeição, orientação por escrito sobre alimentação na gravidez, orientação correta sobre o ganho de peso (procedimentos tipo IV) e orientação para o uso do sulfato ferroso uma hora antes das refeições (procedimento tipo III) foram recebidas por menos de $40 \%$ das gestantes. Tal fato aponta para o despreparo dos profissionais da equipe mínima para lidar com questões nutricionais, que não são específicas de suas profissões.

Em um estudo qualitativo realizado com equipes de saúde da família com o objetivo de apontar as principais dificuldades encontradas pela equipe mínima, os profissionais entrevistados, relataram realizar diversas atividades que não são específicas de suas profissões, devido à falta de profissionais na equipe. Os entrevistados referiram realizar orientação alimentar, através de "dicas" sobre alimentação saudável ${ }^{33}$. Entre os problemas encontrados pela equipe de saúde da família no atendimento à população ressaltam-se a carência de profissionais da equipe mínima ${ }^{23}$, a falta de outros profissionais que não fazem parte dessa equipe $^{33}$ e a dificuldade para encaminhar os pacientes para as especialidades ${ }^{23,33}$, o que leva à não oferta de uma atenção integral à saúde da população atendida pelas equipes de saúde da família ${ }^{23}$.

Ressalta-se que a proporção de relato de recebimento de orientações nutricionais mais específicas não mostrou diferença significativa, ao nível de 5\%, ao comparar os grupos com IMC pré-gestacional adequado e inadequado e com ganho de peso semanal adequado e inadequado, o que pode ter ocorrido devido ao reduzido tamanho amostral. Já a proporção de orientação correta sobre o ganho de peso semanal foi significativamente menor, ao nível de 5\%, entre as gestantes que tiveram ganho de peso semanal inadequado. Preocupa ainda mais a presença de $18,2 \%$ das gestantes que não tinham informações disponíveis para a avaliação do ganho de peso e que possivelmente não receberam uma orientação correta.
Os resultados apontam para uma grande necessidade de assistência nutricional na população atendida pelas sete unidades de saúde da família avaliadas, visando obter melhores desfechos materno-infantis. Ressaltam a necessidade de treinamento da equipe de saúde da família para a valorização e a correta realização dos seguintes procedimentos: aferição e registro das informações antropométricas e de exame físico; avaliação do estado nutricional inicial da gestante, do ganho de peso e do estado nutricional ao longo da gestação; oferta de orientações gerais para as gestantes sobre alimentação saudável; encaminhamento oportuno da gestante para o nutricionista.

A importância dos Núcleos de Apoio à Saúde da Família (NASF), criados pela portaria 154/ $08^{34}$, bem como da inclusão do nutricionista como componente dos mesmos, para a ampliação da abrangência, da integralidade e da resolutibilidade da assistência pré-natal, ganha maior visibilidade neste trabalho. Esse profissional, ao ser inserido em uma estratégia que tem como campos de intervenção o indivíduo, a família e a comunidade, tem a competência necessária, no âmbito da assistência pré-natal, para realizar a promoção de hábitos alimentares saudáveis, prevenção, diagnóstico e tratamento dos distúrbios nutricionais pré-gestacionais (baixo peso, sobrepeso e obesidade), gestacionais (ganho de peso inadequado) e carências nutricionais específicas (anemia e hipovitaminose A); colaborar com a equipe de saúde no cuidado de intercorrências da gestação (hipertensão arterial e diabetes gestacional), educar os componentes da equipe de saúde da família na área de alimentação e nutrição e, como descrito por Assis et al., propor orientações nutricionais adequadas à cultura, às condições fisiológicas e à disponibilidade de alimentos $^{35}$. Os profissionais da equipe mínima e do NASF devem compartilhar seus conhecimentos, permitindo uma visão ampliada da saúde a partir do trabalho multidisciplinar, em direção a uma assistência integral à saúde da população.

Embora este estudo apresente informações que possibilitarão um maior entendimento sobre o processo da assistência nutricional no prénatal em unidades de saúde da família e tenha um papel de subsidiar reflexões e discussões entre profissionais e gestores para a melhoria da qualidade da atenção oferecida, é importante destacar que o mesmo tem uma limitação importante quanto à generalização dos resultados obtidos. Pode-se supor que por conta dos critérios de inclusão empregados para a seleção das 
unidades de saúde da família, as sete unidades participantes ofereçam um atendimento melhor estruturado em comparação com as unidades que não apresentaram estas características.

\section{Colaboradores}

RP Niquini participou da concepção do estudo e foi a responsável principal pela análise e interpretação dos dados, redação do artigo e aprovação final da versão a ser publicada. SA Bittencourt e MC Leal participaram da concepção do estudo, interpretação dos dados, revisão crítica do conteúdo intelectual e aprovação final da versão a ser publicada. EMA Lacerda e C Saunders participaram da interpretação dos dados, revisão crítica do conteúdo intelectual e aprovação final da versão a ser publicada.

\section{Agradecimentos}

Nossos agradecimentos pelo apoio financeiro recebido da Fundação de Amparo à Pesquisa do Estado do Rio de Janeiro (Faperj), do Papes V/ Fiocruz e da Área Técnica de Saúde do Adolescente do Ministério da Saúde.

\section{Referências}

1. Brasil. Ministério da Saúde (MS). Pesquisa Nacional de Demografia e Saúde da Criança e da MulherPNDS 2006: dimensões do processo reprodutivo e da saúde da criança. Brasília: MS; 2009.

2. Nucci LB, Duncan BB, Mengue SS, Branchtein L, Schmidt MI, Fleck ET. Assessment of weight gain during pregnancy in general prenatal care services in Brazil. Cad Saude Publica 2001; 17(6):1367-1374.

3. Padilha PC, Saunders C, Machado RCM, Silva CL, Bull A, Sally EOF, Accioly E. Associação entre o estado nutricional pré-gestacional e a predição do risco de intercorrências gestacionais. Rev Bras Ginecol Obstet 2007; 29(10):511-518.

4. Lacerda EMA, Kac G, Cunha CB, Leal MC. Consumo alimentar na gestação e no pós-parto segundo cor da pele no município do Rio de Janeiro. Rev Saude Publica 2007; 41(6):985-994.

5. Nucci LB, Schmidt MI, Duncan BB, Fuchs SC, Fleck ET, Santos Britto MM. Nutritional status of pregnant women: prevalence and associated pregnancy outcomes. Rev Saude Publica 2001; 35(6):502-507.

6. Baeten JM, Bukusi EA, Lambe M. Pregnancy complications and outcomes among overweight and obese nulliparous women. Am J Public Health 2001; 91(3):436-440.

7. World Health Organization (WHO). Physical status: the use and interpretation of anthropometry: report of a WHO Expert Committee. Geneva: WHO; 1995.

8. Barros DC, Perreira RA, Gama SGN, Leal MC. O consumo alimentar de gestantes adolescentes no município do Rio de Janeiro. Cad Saude Publica 2004; 20(Supl. 1):121-129.

9. Brasil. Ministério da Saúde (MS). Secretaria de Atenção à Saúde. Departamento de Atenção Básica. Saúde da família no Brasil: uma análise de indicadores selecionados: 1998-2005/2006. Brasília: MS; 2008.

10. Brasil. Ministério da Saúde (MS). Secretaria de Atenção à Saúde. Departamento de Ações Programáticas Estratégicas. Estudo da mortalidade de mulheres de 10-49 anos, com ênfase na mortalidade materna Relatório Final. Brasília: MS; 2006.

11. Coutinho T, Teixeira MTB, Dain S, Sayd JD, Coutinho LM. Adequação do processo de assistência pré-natal entre usuárias do Sistema Único de Saúde em Juiz de Fora, MG. Rev Bras Ginecol Obstet 2003; 25(10):717-724. 
12. Carvalho DS, Novaes HMD. Avaliação da implantação de programa de atenção pré-natal no Município de Curitiba, Paraná, Brasil: estudo em coorte de primigestas. Cad Saude Publica 2004; 20(Supl. 2):220-230.

13. Parada CMGL. Avaliação da assistência pré-natal e puerperal desenvolvidas em região do interior do Estado de São Paulo em 2005. Rev Bras Saúde Matern Infant 2008; 8(1):113-124.

14. Silveira DS, Santos IS, Costa JSD. Atenção pré-natal na rede básica: uma avaliação da estrutura e do processo. Cad Saude Publica 2001; 17(1):131-139.

15. Santos LA dos, Mamede FV, Clapis MJ, Bernardi JVB. Orientação nutricional no pré-natal em serviços públicos de saúde no município de Ribeirão Preto: o discurso e a prática assistencial. Rev Latino-am Enferm 2006; 14(5):688-694.

16. Mezzomo CLS, Garcias GL, Sclowitz ML, Sclowitz IT, Brum CB, Fontana T, Unfried RI. Prevenção de defeitos do tubo neural:prevalência do uso da suplementação de ácido fólico e fatores associados em gestantes na cidade de Pelotas, Rio Grande do Sul, Brasil. Cad Saude Publica 2007; 23(11):2716-2726.

17. Donabedian A. The quality of care. How can it be assessed? 1988. Arch Pathol Lab Med 1997; 121(11): 1145-1150.

18. Contandriopoulos AP, Champagne F, Denis JL, Pineault R. A Avaliação na Área de Saúde: Conceitos e Métodos. In: Hartz ZMA, organizador. Avaliação em Saúde. Dos modelos conceituais à prática na análise da implantação de programas. Rio de Janeiro: Fiocruz; 1997. p. 29-47.

19. Scriven M. The Methodology of Evaluation. Social Science Education Consortium 1966; (110):1-58.

20. Brasil. Ministério da Saúde (MS). Secretaria de Atenção à Saúde. Departamento de Ações Programáticas. Pré-natal e Puerpério: atenção qualificada e humanizada - manual técnico. Brasília: MS; 2005.

21. Brasil. Ministério da Saúde (MS). Vigilância alimentar e nutricional (Sisvan): orientações básicas para a coleta, processamento, análise de dados e informação em serviços de saúde. Brasília: MS; 2004.

22. Brasil. Ministério da Saúde (MS). Secretaria de Atenção à Saúde. Departamento de Atenção Básica. Guia alimentar para a população brasileira: promovendo a alimentação saudável. Brasília: MS; 2008.

23. Niquini RP, Bittencourt SA, Lacerda EMA, Saunders C, Leal MC. Avaliação da estrutura de sete unidades de saúde da família para a oferta da assistência nutricional no pré-natal no município do Rio de Janeiro, Brasil. Rev Bras Saúde Matern Infant 2010; 10(Supl. 1):61-68.

24. Niquini RP, Bittencourt SA, Lacerda EMA, Leal MC. Fatores associados à introdução precoce de leite artificial, Município do Rio de Janeiro, 2007. Rev Bras Epidemiol 2009; 12(3):446-457.
25. Atalah ES, Castillo CL, Castro RS, Aldea AP. Propuesta de un nuevo estándar de evaluación nutricional en embarazadas. Rev. Méd. Chile 1997; 125(12): 1429-1436.

26. Institute of Medicine, National Academy of Sciences. Nutrition During Pregnancy and Lactation: An Implementation Guide. Washington DC: National Academy Press; 1992.

27. Institute of Medicine, National Academy of Sciences. Nutrition during Pregnancy. Washington DC: National Academy Press; 1990.

28. Saunders C, Bessa TCCA, Padilha PC. Assistência Nutricional Pré-natal. In: Accioly E, Saunders C, Lacerda EMA, organizadores. Nutrição em Obstetrícia e Pediatria. Rio de Janeiro: Guanabara Koogan; 2009. p. 103-124.

29. Byrt T, Bishop J, Carlin JB. Bias, Prevalence and Kappa. J Clin Epidemiol 1993; 46(5):423-429.

30. Landis JR, Koch GG. The measurement of observer agreement for categorical data. Biometrics 1977; 33(1):159-174.

31. Costa GD, Cotta RMM, Reis JR, Siqueira-Batista R, Gomes AP, Franceschini SCC. Avaliação do cuidado à saúde da gestante no contexto do Programa Saúde da Família. Cien Saude Colet 2009; 14 (Supl. 1):1347-1357.

32. Brasil. Decreto no 5.749 de 11 de abril de 2006. Altera o caput do art. 18 do Decreto no 5.209 , de 17 de setembro de 2004, dispondo sobre atualizações de valores referenciais para caracterização das situações de pobreza e extrema pobreza no âmbito do Programa Bolsa Família. Diário Oficial da União 2006; 12 abr.

33. Loch-Neckel G, Seemann G, Eidt HB, Rabuske MM, Crepaldi MA. Desafios para a ação interdisciplinar na atenção básica: implicações relativas à composição das equipes de saúde da família. Cien Saude Colet 2009; 14(Supl. 1):1463-1472.

34. Brasil. Portaria no 154 de 24 de janeiro de 2008. Cria os Núcleos de Apoio à Saúde da Família - NASF. Diário Oficial da União 2008; 25 jan.

35. Assis AMO, Santos SMC, Freitas MCS, Santos JM, Silva MCM. O Programa Saúde da Família: contribuições para uma reflexão sobre a inserção do nutricionista na equipe multidisciplinar. Rev Nutr Campinas 2002; 15(3):255-266.

Artigo apresentado em 03/02/2011

Aprovado em 10/05/2011

Versão final apresentada em 26/06/2011 